\title{
La vigencia de las armas nucleares en el siglo XXI
}

\section{The Relevance of Nuclear Weapons in the 21 st Century}

\author{
Carlos Javier Frías Sánchez ${ }^{1}$ \\ Coronel del Ejército de Tierra (España)
}

Recibido: 01-05-20

Aprobado: 06-06-20

\section{Resumen}

El mundo está en proceso de cambio. El sistema internacional ha pasado de una estructura bipolar a otra unipolar, y evoluciona hoy hacia una multipolar. Sin embargo, la actual "multipolaridad es desequilibrada" y, por ello, inestable, con una superpotencia (Estados Unidos) luchando por mantener su ventaja relativa frente a sus rivales, otra cuya principal baza es su arsenal nuclear (Rusia) y una tercera que busca recuperar el papel de hegemón al que cree ser acreedor (China). Por otra parte, el actual régimen de no proliferación nuclear es "hijo" de la Guerra Fría y su diseño responde a la distribución de poder y tecnología existente en el momento de su creación. Conforme pasa el tiempo, y estas condiciones se apartan más de las que permitieron su creación, el régimen está peor adaptado a sus fines. En consecuencia, existen muchas posibilidades de una reactivación de la proliferación nuclear en el futuro.

Palabras-clave: Régimen, proliferación nuclear, sistema internacional, multipolaridad desequilibrada, armas nucleares.

\footnotetext{
${ }^{1}$ (cfriassanchez@gmail.com ) Carlos Javier Frías Sánchez nació en Madrid en 1967. Es coronel del Ejército de Tierra, actualmente destinado como jefe del Regimiento de Artillería Antiaérea ${ }^{\circ} 73$ con guarnición en Cartagena (Murcia). Es diplomado de Estado Mayor de las Fuerzas Armadas (2005), máster en Estudios Estratégicos por la Universidad de Granada (2011), máster en Seguridad y Defensa por el CESEDEN y la Universidad Complutense (2012) y doctor en Paz y Seguridad Internacionales por el Instituto Universitario General Gutiérrez Mellado (2015). Ha tenido numerosos destinos en España y en el extranjero, y ha efectuado misiones en los Balcanes y en África. Es autor de varios artículos y publicaciones en revistas españolas y extranjeras sobre temas militares (especialmente en los campos de doctrina e historia militar) y estratégicos (especialmente sobre disuasión y armamento nuclear).
} 


\begin{abstract}
The world is changing. Our international system is evolving from a bipolar structure to a unipolar one and is moving towards a multipolarity. However, this multipolarity is unbalanced, and hence, unstable, with a Superpower struggling to keep its rivals at bay with decreasing resources (USA), another one whose only trump card is its nuclear arsenal (Russia) and a third one seeking to recover its ancient hegemonic role (China). On the other hand, the current nuclear non-proliferation regime is a legacy from the Cold War, and its design and operation are based on the distribution of power and technology at the time of its inception. As time passes and this distribution is more and more different from the one it was based on, this regime is less and less apt to achieve their pretended goals. Therefore, there is a high possibility of a re-activation of nuclear proliferation processes soon.
\end{abstract}

Key-words: Regime, Nuclear Proliferation, International System, Unbalanced Multipolarity, Nuclear Weapons.

"El viejo mundo se muere, el nuevo tarda en aparecer, y en ese claroscuro surgen los monstruos"

Antonio Gramsci

\title{
Introducción
}

Resulta un tópico mencionar que el final de la Guerra Fría supone un cambio fundamental en el panorama estratégico que ha estado vigente desde el final de la Segunda Guerra Mundial.

La distribución de poder entre los Estados tras la Segunda Guerra Mundial y la particular estructura del sistema internacional durante la Guerra Fría configuraron una serie de instituciones que han dado forma a nuestro mundo. Para varias generaciones, la existencia de estas instituciones era una parte integrante de la forma de funcionar de nuestro mundo. Así, la Organización de Naciones Unidas, el Acuerdo General sobre Aranceles y Comercio (GATT, por sus siglas en inglés) de 1947, la Organización Mundial del Comercio, el Banco Mundial, la Organización del Tratado del Atlántico Norte y el Pacto de Varsovia, entre otros, eran las instituciones que proporcionaban el marco en el que se desenvolvían las relaciones entre los Estados. Uno de estos elementos que configuraban el mundo durante la Guerra Fría era el régimen de no 
proliferación nuclear, el conjunto de instituciones e instrumentos jurídicos que tenían como finalidad impedir la extensión de las armas nucleares.

Hasta tal punto estos instrumentos e instituciones se convirtieron en omnipresentes que, en cierta manera, nos hicieron olvidar que su origen y permanencia estaban ligadas a la continuidad de un determinado reparto de poder.

La brusca caída de la Unión Soviética supuso un terremoto geopolítico: de la división del mundo en dos bloques rivales, encabezados cada uno por una superpotencia, pasamos a un mundo en que Estados Unidos superaba ampliamente en poder y en influencia a cualquier otro Estado del sistema internacional.

El hecho de que Estados Unidos -y sus valores- fuera el inspirador de la mayoría de las instituciones creadas tras la Segunda Guerra Mundial, supuso la supervivencia de muchas de ellas (de todas aquellas ligadas a la instauración de un "orden liberal" basado en el libre comercio y la extensión progresiva de la democracia liberal), el declive de otras que "necesitaban" la rivalidad entre las dos superpotencias para justificar su actividad (como la OTAN o, en menor medida, la ONU) y la desaparición de aquellas ligadas directamente a la superpotencia desaparecida (como el Pacto de Varsovia o el COMECON).

La permanencia de muchas de estas organizaciones y la "inercia institucional" de todas ellas han ocultado hasta cierto punto el hecho de que la situación del mundo ha cambiado y que el marco que estas instituciones daban a las relaciones internacionales no corresponde a la actual estructura del sistema internacional. Y, cuanto más nos alejamos del periodo en que estas instituciones eran válidas -la Guerra Fría-, mayor es la inadecuación entre ellas y la realidad.

En el campo del armamento nuclear, el régimen de no proliferación nuclear es uno de estos casos de declive. La abrogación del Tratado sobre Misiles Antibalísticos (también llamado ABM Treaty, firmado en 1972 y denunciado en 2002), así como la del Tratado sobre Fuerzas Nucleares de Alcance Intermedio (o INF Treaty, firmado en 1987 y abandonado en 2019) son dos ejemplos del declive del conjunto del régimen.

¿Quiere esto decir que estas instituciones deben desaparecer? En ningún caso. Son instrumentos que han proporcionado seguridad y prosperidad durante muchos años, pero es imperativo adaptarlos a la estructura real del mundo actual, que dista mucho de la existente en el momento de su creación. 


\section{"EI viejo mundo" (nuclear)}

La aparición del arma nuclear en 1945 supuso más que un terremoto geopolítico: forzó un cambio de paradigma que cuestionaba la propia naturaleza del fenómeno de la guerra, una de las constantes que han acompañado indefectiblemente a la humanidad desde sus mismos albores.

Para entender la dimensión del cambio que implicó el arma nuclear es útil recordar alguna de las citas más relevantes que "dieron la bienvenida" al nuevo ingenio. Así, Bernard Brodie ${ }^{2}$ citaba, en los albores de la era nuclear, que “... hasta el momento presente el propósito principal de nuestro sistema militar ha sido ganar guerras. De ahora en adelante, su propósito principal debe ser evitarlas. No puede tener prácticamente ningún otro propósito".

En efecto, la aparición del arma nuclear configuró un mundo que disfrutaba de una paz (relativa), pero que vivía bajo la amenaza perenne de un "Armagedón nuclear". La presencia de las armas nucleares era un factor permanente que dominaba las relaciones internacionales, y la opinión pública en todo el mundo era agudamente consciente de las posibles consecuencias de una (siempre posible) guerra nuclear. La sombra de la disuasión nuclear afectaba de forma determinante a todas las crisis y a todos los conflictos, incluso locales.

Además, las armas nucleares son elementos fundamentales de la distribución de poder en el sistema y condicionan las relaciones de disuasión entre los Estados ${ }^{3}$, lo que evidencia la relación directa entre la estructura del sistema internacional y el armamento nuclear.

Las armas nucleares configuraron un mundo "único", sin precedentes históricos y muy particular, distinto del mundo anterior a la Guerra Fría y del que la sucedió.

\section{Un mundo "bipolar"}

La estructura del sistema internacional en un momento determinado queda determinada por el número de "polos" existentes entonces. Según su número, esta estructura puede ser unipolar (un solo polo dominante), bipolar (dos polos) o multipolar (varios polos).

\footnotetext{
${ }^{2}$ Bernard S. Brodie, The Absolute Weapon: Atomic Power and World Order, Nueva York, Harcourt Brace, 1946, p. 76.

3 Clark A. Murdock, "A Nuclear Deterrent for the 21st Century", en Craig Cohen y Josiane Gabel, en Global Forecast 2015, Crisis and Opportunity, Washington, Center for Strategic and International Studies, 2014, p. 17. Disponible en: http://csis.org/files/publication/141110_Cohen GlobalForecast2015 Web.pdf
} 
Para Snyder y Diesing4, el número de "polos" de poder del sistema internacional lo fija el número de "actores principales" presentes en cada momento en el seno del sistema y "la distribución de poder militar", entre ellos: en un sistema "bipolar", existen dos grandes potencias cuyo poder militar es similar y cuya rivalidad domina las interacciones entre los componentes del sistema. En esta aproximación "realista", el poder se mide esencialmente en términos de "poder militar".

En una aproximación más amplia, Kenneth N. Waltz ${ }^{5}$ considera que el poder de los Estados puede medirse en varias esferas diferentes (económica, militar, política y demográfica) y que la consideración de gran potencia depende del poder agregado del Estado, es decir, de la suma de su tamaño físico, población, dotación de recursos, fuerza militar, estabilidad política y competencia.

La aparición del arma nuclear y el hecho de que solo Estados Unidos y la Unión Soviética estuviesen en condiciones de producir arsenales nucleares masivos determinó una nueva estructura del sistema internacional. En efecto, la posesión de enormes arsenales nucleares en manos de estas dos superpotencias hizo que ningún otro Estado pudiera rivalizar de ninguna manera con ninguna de ellas, ni pudiera tener ninguna esperanza de éxito en un conflicto militar con cualquiera de ellas, independientemente de la competencia de sus Fuerzas Armadas. De esta manera, el sistema internacional se configuraba como una estructura con dos "polos", dos superpotencias rivales entre sí, mientras que ningún otro miembro del sistema internacional se acercaba remotamente al poder (agregado o simplemente militar) de cada una de ellas. La estructura del sistema internacional durante la Guerra Fría respondía en consecuencia al modelo de la bipolaridad.

En términos históricos, la estructura bipolar no es particularmente estable. Los casos conocidos (Roma y Cartago o Atenas y Esparta) ${ }^{6}$ se caracterizaron precisamente por una situación de conflicto casi permanente. Sin embargo, la Guerra Fría fue un conflicto relativamente "incruento", en el sentido de que ambas superpotencias eligieron dirimir sus diferencias a través de sus Estados $\mathrm{u}$ organizaciones aliadas. Las razones de esto hay que buscarlas precisamente en el efecto de las armas nucleares ${ }^{7}$.

${ }^{4}$, Glenn H. Snyder y Paul Diesing, Conflict among Nations: Bargaining, Decision Making and System Structure in International Crisis, Princeton, Princeton University Press, 1977, pp. 419-420.

${ }^{5}$ Kenneth N. Waltz, Theory of International Politics, Long Grove, Waveland Press Inc., 2010, pp. 88-99.

${ }^{6}$ Karl W. Deutsch y David J. Singer. "Multipolar Power Systems and International Stability", en World Politics, de la Universidad John Hopkins, vol. 16, nº 3, de abril de 1964, pp. 390-406, p. 406.

7 Alfred Wohlstetter, "The Delicate Balance of Terror", Santa Monica, RAND Corporation, 6 de noviembre de 1958, accesible en la página web de la RAND Corporation, en http://www.rand.org/ about/history/wohlstetter/P1472/P1472.html 20 de mayo de 2019.

Araucaria. Revista Iberoamericana de Filosofia, Política, Humanidades y Relaciones Internacionales, año $22, \mathrm{n}^{\circ} 44$. Segundo semestre de 2020. Pp. 427-448. ISSN 1575-6823 e-ISSN 2340-2199 https://dx.doi.org/10.12795/araucaria.2020.i44.20 
La estructura bipolar se caracteriza además porque obliga a los Estados a alinearse con uno u otro de los polos sin posibilidad de permanecer al margen de la rivalidad entre las dos superpotencias, como muestra crudamente "el diálogo de melios" que recoge Tucídides en La Guerra del Peloponeso. Este efecto se materializa en la aparición de dos bloques rivales. Una consecuencia de ello es que las rivalidades entre Estados "menores" del sistema acaban atrayendo la atención de las dos superpotencias y los conflictos entre estos Estados "menores" acaban siendo parte de la pugna más amplia entre las superpotencias. Las guerras árabe-israelíes son una muestra de este fenómeno. El conflicto entre Israel y sus vecinos árabes por el dominio de Palestina acabó convertido en una guerra en la que Israel era un aliado de Estados Unidos y los países árabes lo eran de la Unión Soviética. Como consecuencia, ambas superpotencias enviaron gran cantidad de armamento a sus aliados respectivos. De la misma forma, los armisticios que siguieron a cada uno de los episodios de ese largo conflicto fueron en realidad acuerdos alcanzados por ambas superpotencias e impuestos a sus aliados.

Un tercer efecto de la bipolaridad es que las superpotencias son capaces de ejercer una enorme presión sobre sus aliados ante la amenaza de abandonarlos a su suerte frente al poder aplastante de la superpotencia rival.

Armas de fisión y armas de fusión

En realidad, aunque se hable genéricamente de "armas nucleares", existen dos tipos principales de armas nucleares, atendiendo al principio físico que explica su funcionamiento: las armas de fisión (también llamadas atómicas) y las armas de fusión (también conocidas como termonucleares o de hidrógeno). Estos dos tipos de armas tienen diferencias suficientes como para tenerlas muy presentes en todas las discusiones sobre este tipo de armamento.

Las armas de fisión basan su funcionamiento en conseguir la escisión de los núcleos de átomos de elementos pesados (uranio o plutonio) que pasan a convertirse en átomos de elementos más ligeros, generando gran cantidad de energía. Las armas de fusión intentan conseguir unir núcleos de elementos ligeros (en general, hidrógeno) que se convierten en elementos más pesados (helio, por ejemplo). La energía necesaria para conseguir la fusión de los núcleos de hidrógeno es enorme, tan grande que se emplea una bomba de fisión para proporcionar esta energía, pero la fusión genera una cantidad de energía varios órdenes de magnitud superior. En consecuencia, las armas de fusión son miles de veces más potentes que las de fisión: mientras que estas últimas miden su potencia en kilotones (miles de toneladas de trinitrotolueno, el explosivo militar más habitual), las de fusión llegan a las decenas de megatones (millones de toneladas de trinitrotolueno), como fue el caso de la Bomba del Zar que alcanzó los 57 megatones en el ensayo que la Unión Soviética realizó en Nueva Zembla el 30 de octubre de 1961. A modo de comparación, la bomba lanzada 
sobre Hiroshima tenía una potencia de unos 13 kilotones y la que cayó sobre Nagasaki alcanzó los 21 kilotones.

La diferencia de nivel tecnológico necesario para producir armas nucleares de fisión y armas nucleares de fusión es muy importante. La tecnología de la bomba de fisión es relativamente sencilla, además de que se han producido importantes filtraciones tecnológicas que han allanado las dificultades técnicas para construir este tipo de $\operatorname{armas}^{8}$, de forma que la principal dificultad para su fabricación es el acceso a determinados materiales (fundamentalmente, el material fisible, sea uranio enriquecido o sea plutonio). La fabricación de la bomba de hidrógeno supone el dominio previo de la tecnología de la bomba de fisión ${ }^{9}$ seguida de un salto tecnológico importante y complejo ${ }^{10}$ para obtener la bomba de fusión.

Las características de las armas termonucleares las hacen en realidad muy diferentes de las armas de fisión. En palabras de Churchill11 "Hay una inmensa distancia entre la bomba atómica y la de hidrógeno. La bomba atómica, con todo su horror, no nos lleva fuera de los límites del control humano, o de los acontecimientos manejables en pensamiento o en acción, en paz o en guerra. Pero... [con] la bomba de hidrógeno, los fundamentos de las cuestiones humanas sufrieron una revolución".

En efecto, como explica Mandelbaum", "Ninguna [de las dos bombas atómicas lanzadas sobre Japón] causó tantos daños como los que provocaron las incursiones aéreas que los Estados Unidos habían llevado a cabo con explosivos no nucleares contra Tokio, Yokohama y Dresde".

Esta diferencia es más importante de lo que pueda parecer, puesto que la producción de armas de fusión es un paso posterior y tecnológicamente mucho más complejo que la fabricación de armas de fisión con excepción de Estados Unidos, Rusia, China, Francia y el Reino Unido, ningún otro Estado dispone de armas de fusión. Los casos de proliferación nuclear más "mediáticos" (Irán o Corea del Norte) se centran actualmente en la producción de armas de fisión (aunque los norcoreanos alardearon de haber conseguido una explosión termonuclear en septiembre de 2017, pero existen fundadas dudas de

\footnotetext{
${ }^{8}$ La red del paquistaní A. Q. Khan distribuyó el diseño de una cabeza nuclear de fisión china apta para su empleo en misiles nucleares; el OIEA descubrió y mostró el diseño de una cabeza nuclear de fisión apta para su empleo en manos de Saddam Hussein. Citado en Henry D. Sokolski (ed.), The Next Arms Race, Carlisle Barracks, Strategic Studies Institute, julio de 2012, p. 24, accesible en http://www.strategicstudiesinstitute.army.mil/pdffiles/PUB1113.pdf, 8 de septiembre de 2019.

${ }_{9}$ Bertrand Goldschmdt, The Atomic Complex. A Worldwide Political History of Nuclear Energy. La Grange Park, American Nuclear Society, 1982, p. 104.

10 Aunque tal vez menos complejo de lo esperable, debido a una deficiente preservación de las tecnologías necesarias. Ibid, p. 211.

11 Michael Mandelbaum, The Nuclear Revolution: International politics before and after Hiroshima, Cambridge, University of Cambridge Press, 1981, p. 3.

12 Ibid, p. 2.
} 
este hecho) ${ }^{13}$. Y, como decían Churchill y Mandelbaum, lamentablemente, la humanidad tiene experiencia en guerras donde se han empleado profusamente armas capaces de destruir una ciudad en un día ${ }^{14}$.

Sin embargo, esto no es completamente cierto. La fisión nuclear necesita reunir una masa de algún elemento radiactivo, cuyos núcleos atómicos emiten neutrones de forma espontánea. Esos neutrones pueden chocar con otros núcleos, rompiéndolos y produciendo nuevas emisiones de neutrones. Sin embargo, en estado natural, esta actividad radioactiva produce energía, pero el número de colisiones de neutrones es tan pequeño que esa energía es casi imperceptible. Para aumentar el número de colisiones de neutrones se "enriquece" el material radiactivo: el uranio existe en dos isótopos en la naturaleza, el uranio-238 (97\% del existente) y uranio-235 (3\% restante) que coexisten mezclados. El uranio-238 es mucho más estable (emite menos neutrones), mientras que el uranio-235 es más activo.

El "enriquecimiento" consiste en separar el uranio-238 del uranio-235, reservando este último para producir material fisible. Por su parte, el plutonio es un elemento artificial, altamente radiactivo que consiste en un subproducto de la fisión del uranio, lo que implica que la producción de plutonio implica disponer de un reactor nuclear de uranio. La fisión "explosiva" se produce cuando se reúne una cierta cantidad de uranio "enriquecido" o plutonio, cantidad llamada "masa crítica" (diferente para cada uno de estos elementos).

En ese momento, la emisión de neutrones es suficiente para que se produzca una reacción en cadena: los neutrones emitidos chocan con núcleos de átomos, los rompen y estos núcleos rotos emiten nuevos neutrones que alcanzan a otros núcleos, rompiéndolos a su vez... Las bombas de fisión mantienen separada la masa crítica de uranio o plutonio en cantidades menores (llamadas "masas subcríticas"), reuniéndolas rápidamente en el momento de la explosión. Sin embargo, la explosión disgrega la masa crítica, por lo que es necesario forzar al máximo el tiempo en que la masa crítica se encuentra reunida. Esto se hace envolviendo las masas subcríticas en una estructura construida de un material lo más resistente posible (tungsteno) o mediante lentes explosivas (bombas de implosión). Incluso así, la cantidad de material radiactivo que se consume en la explosión es muy pequeña (del orden del 2 o el $3 \%$ ). El resto de este material altamente radiactivo se dispersa con la explosión.

En consecuencia, las armas nucleares producen efectos no solo térmicos y mecánicos (como cualquier otra bomba), sino que además contaminan radiactivamente amplias áreas, imposibilitándolas para la vida humana durante

\footnotetext{
${ }^{13}$ Robert Windrem, "North Korea likely lying about Hydrogen bomb test, experts say", en $N B C$ News.

${ }^{14}$ Franklin D’Olier, et alt., U.S. Strategic Bombing Survey, Washington, 1946 (reimpreso en 1987 por la USAF). Disponible en: http://web.archive.org/web/20080528051903/http://aupress.au.af.mil/ Books/USSB S/USSBS.pdf.
}

Araucaria. Revista Iberoamericana de Filosofia, Política, Humanidades y Relaciones Internacionales, año $22, \mathrm{n}^{\circ} 44$. Segundo semestre de 2020. Pp. 427-448. ISSN 1575-6823 e-ISSN 2340-2199 https://dx.doi.org/10.12795/araucaria.2020.i44.20 
largos periodos de tiempo. Es decir, sus efectos exceden con mucho los de los bombardeos convencionales.

Sin embargo, la rápida aparición del arma termonuclear (la primera explosión de estas armas se produjo en 1952) y la evolución de la estrategia nuclear de las dos superpotencias, basada cada vez más en las bombas de hidrógeno, hicieron que las armas de fisión pasasen casi desapercibidas en comparación con la amenaza que suponían las armas de fusión.

\section{La "destrucción mutua asegurada"}

Quizá el concepto más conocido de la estrategia nuclear de la Guerra Fría sea el de la "destrucción mutua asegurada" (MAD, por sus siglas en inglés). Las connotaciones apocalípticas que invoca esta expresión hicieron que la MAD fuera una especie de resumen de lo que significaría una guerra en la que se empleasen armas nucleares. Solo podría acabar con la extinción del género humano o, incluso, de la vida en el planeta. Sin embargo, la MAD fue la "etapa final" del desarrollo de una serie de ideas sobre la forma de emplear las armas nucleares (o, mejor dicho, de evitar su uso).

Si en los primeros momentos de la era nuclear, el monopolio norteamericano de esta tecnología parecía apuntar hacia una situación de unipolaridad, la adquisición por la Unión Soviética de la tecnología necesaria para desarrollar armas nucleares obligó a ambas superpotencias a reflexionar sobre la utilidad y el posible empleo de las armas nucleares.

En realidad, aunque ya desde el primer momento de su aparición algunos autores consideraron estas novedosas armas como algo fundamentalmente distinto del resto de los medios militares (denominados, por exclusión, “convencionales"), en los primeros años tras la aparición de las armas nucleares había muchos especialistas que consideraban el arma nuclear simplemente como una bomba convencional, pero con una potencia extraordinaria. Consecuentemente, se desarrollaron armas destinadas al empleo en combate de estas armas (como los “cañones atómicos") y se esbozaron doctrinas de empleo del armamento atómico.

Sin embargo, el desarrollo de las armas termonucleares (Estados Unidos las probó ya en 1952, mientras que la Unión Soviética detonó su primera bomba de hidrógeno al año siguiente, en 1953) llevó al convencimiento que expresaba la mencionada cita de Churchill: las armas termonucleares eran algo substancialmente distinto de las armas convencionales. En efecto, el nivel de destrucción que podrían causar estas armas era tal que requerían una aproximación intelectual diferente. 
En 1954, Alfred Wohlstetter realizó un detallado estudio sobre la vulnerabilidad de las bases aéreas norteamericanas (en aquel momento, el único vector de lanzamiento de armas nucleares eran los bombarderos pesados) ante un ataque aéreo soviético ${ }^{15}$. Wohlstetter apuntaba así uno de los primeros conceptos clave de la estrategia nuclear de la Guerra Fría: la capacidad del "primer golpe" (first strike), entendida como la posibilidad de emplear armas nucleares contra un adversario con la finalidad de destruir su arsenal nuclear antes de que pueda emplearlo. Es importante tener en cuenta que la definición de "capacidad del primer golpe" se realizó en los primeros años de la Guerra Fría cuando la extensión geográfica de ambas superpotencias y la imprecisión de los sistemas de ataque (factores a los que se unieron prontamente la dispersión de los arsenales nucleares y las medidas de protección física de las instalaciones ligadas a estos arsenales) determinaron que la capacidad de destruir el armamento nuclear del enemigo solo era alcanzable por medio de ataques con armamento nuclear.

Como consecuencia lógica de la posibilidad de sufrir un "primer golpe", aparece el concepto de "segundo golpe" (second strike), la capacidad de responder a un ataque de "primer golpe" con armamento nuclear capaz de causar un daño inaceptable al enemigo. Los trabajos del Comité Gaither, organizado por el Gobierno norteamericano en 1957 y encargado de estudiar la estrategia a seguir con el armamento nuclear, concluyeron que la disuasión basada en la amenaza mutua dependía de la seguridad de que la fuerza encargada de la represalia pudiera sobrevivir a un primer ataque enemigo ${ }^{16}$. Es decir, que la adquisición y el mantenimiento de la capacidad de "segundo golpe" era una parte imprescindible de la disuasión nuclear.

A partir de los conceptos de "primer golpe" y "segundo golpe", la evolución de los arsenales nucleares era previsible. Aunque ninguna de las dos superpotencias admitiese que sería la primera en lanzar un "primer golpe" sobre su rival, ambas desconfiaban de la otra. Para evitar sufrir un ataque del "primer golpe" con éxito, era necesario tener un arsenal nuclear tan grande como para que una parte de él pudiera sobrevivir para asestar un "segundo golpe". Sin embargo, cuanto más crecía el arsenal nuclear enemigo (y, puesto de que las armas de "primer golpe" eran indistinguibles de las de "segundo golpe"), mayor era su capacidad de asestar un "primer golpe" eficaz. Así, a partir de los años 60, las dos superpotencias se embarcaron en una vertiginosa

\footnotetext{
${ }^{15}$ Alfred Wohlstetter et alt., Selection and Use of Strategic Air Bases, Santa Monica, RAND Corp. United States Air Force project RAND R-266, de abril de 1954 (reimpreso en 1962), accesible en http://www.rand.org/content/dam/rand/pubs/reports/2006/R266.pdf 18 de febrero de 2020.

16 Thomas C. Schelling, en Elbridge A. Colby y Michael S. Gerson, Strategic Stability: Contending Interpretation, Carlisle, Strategic Studies Institute, febrero de 2013, p. v, accesible en http://www. strategicstudiesinstitute.army.mil/pdffiles/PUB1144.pdf, 12 de febrero de 2020.
} 
carrera de armamento nuclear que llevó a que su capacidad destructiva fuese suficiente como para acabar con el planeta varias veces.

Como consecuencia, a principios de los años 60, el secretario de Estado del presidente norteamericano Kennedy, Robert S. McNamara, enunciaba la teoría de la "destrucción mutua asegurada" (MAD, por sus siglas en inglés), entendida como la situación en la que un conflicto nuclear entre las dos superpotencias implicaría inevitablemente la destrucción de ambas. En palabras del propio McNamara en 1967, "Es importante comprender que la destrucción asegurada es la quintaesencia de todo el concepto de disuasión". El concepto fue duradero: en julio de 1980, el secretario de Defensa norteamericano, Harold Brown, declaró: "lo que ha llegado a ser conocido como destrucción asegurada es el cimiento de la disuasión nuclear, y mantendremos tal capacidad en el futuro" ${ }^{17}$. Es importante citar que McNamara no enunciaba una "teoría", sino que simplemente comunicaba que la estrategia nuclear norteamericana se basaría en un hecho incontrovertible: una guerra nuclear implicaría la destrucción de ambas superpotencias, y con ellas, de la vida en la Tierra.

Un fenómeno derivado de la MAD fue lo que Glenn Snyder llamó la "paradoja de la estabilidad-inestabilidad"18: la estabilidad nuclear derivada de existencia de la MAD se tradujo en la aparición de múltiples conflictos "menores", de tipo no nuclear, puesto que las dos superpotencias tenían la garantía de que la otra no recurriría a una represalia que pudiera conducir al estallido de una guerra nuclear total.

Dado el alcance global de la destrucción originada en caso de una guerra nuclear entre las superpotencias, el mantenimiento de la paz entre ambas superpotencias era del máximo interés de todos los Estados (y de todos sus habitantes), pues nadie podría quedar al margen de las catastróficas consecuencias de una guerra termonuclear.

Sin embargo, a veces tendemos a olvidar que la MAD implica el intercambio de miles de cabezas termonucleares, algo que solo estaba al alcance de las Superpotencias de la Guerra Fría. El nivel de destrucción que acarrearía una guerra nuclear "limitada" entre Estados con armas de fisión distaría mucho del esperado en la temida MAD y, según el área geográfica que abarcase el conflicto, habría Estados más afectados por él, mientras que otros sufrirían las consecuencias en menor medida. En consecuencia, para según qué posibles diadas de conflicto nuclear, no todos los Estados sentirían que es de su máximo interés evitar ese conflicto. De la misma manera, sería posible que algún Estado (incluso nuclear) apoyase a grupos radicales capaces de emplear una de estas armas nucleares "limitadas".

${ }^{17}$ Citado en John T. Correll, "The Ups and Downs of Counterforce", en Air Force Magazine, octubre de 2005, pp. 58-64, pp. 61 y 63.

${ }^{18}$ Glenn Snyder, "The Balance of Power and the Balance of Terror," en, Paul Seabury (ed.), The Balance of Power, San Francisco, Chandler, 1965, pp. 184-201.

Araucaria. Revista Iberoamericana de Filosofí, Política, Humanidades y Relaciones Internacionales, año 22, $\mathrm{n}^{\circ} 44$. Segundo semestre de 2020. Pp. 427-448. ISSN 1575-6823 e-ISSN 2340-2199 https://dx.doi.org/10.12795/araucaria.2020.i44.20 


\section{El régimen de la no proliferación nuclear}

Como se ha citado, uno de los efectos del alineamiento de los Estados en bloques, propio de la Guerra Fría, es que todos los conflictos, incluso locales, acababan subsumidos en la gran rivalidad entre las dos superpotencias. Cuando esos conflictos aparecían entre Estados del mismo bloque, la superpotencia hegemónica dentro del bloque afectado acababa arbitrando una solución más o menos justa y más o menos duradera. Sin embargo, cuando afectaba a Estados rivales alineados en diferentes bloques, el riesgo de que un conflicto local acabase escalando a un conflicto mayor entre las dos superpotencias era elevado. Si no había armas nucleares por medio, la citada paradoja de la estabilidadinestabilidad hacía que resultase en un conflicto más o menos intenso, pero siempre limitado. En cambio, si existía la posibilidad de que alguno de los contendientes llegase a emplear armas nucleares, el riesgo de una escalada que degenerase en una guerra termonuclear entre ambas superpotencias era muy elevado.

La necesidad de reducir en lo posible el riesgo de una guerra termonuclear llevó a la creación del conjunto de acuerdos e instituciones conocido como el régimen de no proliferación nuclear, un conjunto de instrumentos jurídicos y políticos destinados a evitar que nuevos Estados accediesen al arma nuclear y (en teoría) a alcanzar el desarme de los Estados que ya poseían ese armamento.

El régimen de no proliferación nuclear es precisamente un "régimen", y más concretamente un "régimen de seguridad". En una definición ampliamente aceptada, para Krasner, los regímenes son ${ }^{19}$ "un conjunto de principios, normas, reglas y procedimientos de tomas de decisiones explícitos o implícitos alrededor de los cuales convergen las expectativas de los actores en áreas específicas de las Relaciones Internacionales".

Krasner fija como factor que justifica la creación de un régimen la existencia de principios y normas compartidas. Para los regímenes de seguridad, Jervis añade que el mantenimiento de los regímenes de seguridad depende bien de una imposición hegemónica o bien precisa que los Estados participantes en ellos otorguen la misma importancia a los "valores de seguridad mutua y cooperación" 20 .

En realidad, con relación a la creación y mantenimiento de los regímenes internacionales, los estudiosos mantienen básicamente tres posiciones:

- El realismo en sus diferentes tendencias, que defiende que los regímenes son un epifenómeno, un reflejo de las relaciones de poder existentes en el sistema internacional en cada momento. Como

${ }_{19}$ Stephen D. Krasner, International Regimes, Ithaca, Cornell University Press, 1982, p. 2.

20 Jervis, Robert, "Security regimes", en S. Krasner, International Regimes, op. cit., p. 177. 
consecuencia, no tienen un efecto real, más allá de ser vehículos de los Estados poderosos para hacer avanzar sus intereses ${ }^{21}$.

- La escuela liberal, que considera que los regímenes son una característica constante e inherente al sistema internacional: cualquier conducta pautada da lugar a un régimen (formal o informal) $\mathrm{y}$, por lo tanto, tienen influencia en muchas facetas de las relaciones internacionales, ya sea mediante una estructura formal o por uso no reglado. En esta corriente de pensamiento, la mayor interconexión entre los diferentes Estados es un factor que favorecería la aparición $\mathrm{y}$ el fortalecimiento de los regímenes ${ }^{22}$.

- Una posición intermedia defiende que los regímenes están determinados por las relaciones de poder, pero que, en circunstancias concretas, estos regímenes influyen en el comportamiento de los Estados $^{23}$.

En cualquier caso, la situación particular de la Guerra Fría favorecía la creación de un instrumento que redujese el riesgo de una guerra termonuclear entre ambas superpotencias. Como consecuencia, la práctica totalidad de los Estados aceptaron la creación de una serie de instrumentos jurídicos que restringían su soberanía, al tiempo que garantizaba unos derechos diferentes a un reducido grupo de Estados. En efecto, el elemento clave del régimen, el Tratado de No Proliferación Nuclear (TNP) de 1968, otorga a cinco Estados (Estados Unidos, la Unión Soviética, China, Francia y el Reino Unido) el estatus de "Estados nucleares" con el derecho de poseer armas nucleares, derecho al que renuncian el resto de los Estados signatarios del tratado.

En su primer párrafo, el TNP alude como razón de su redacción a "la devastación que causaría una guerra nuclear". Sin embargo, su funcionamiento se basa en tres compromisos interrelacionados de carácter mucho más práctico que moral:

-Por un lado, pretende reducir los incentivos de los Estados para hacerse con armamento nuclear, al garantizar que tampoco sus rivales obtendrán estas armas, evitando así las "carreras de armamentos" nucleares.

-Por otro, ofrece ayuda técnica para aprovechar los usos pacíficos de la energía nuclear, sujeta a la renuncia al armamento nuclear. -Finalmente, ofrece la posibilidad del desarme nuclear de los Estados nucleares, es decir, promete el final del riesgo de una guerra nuclear que afectase a todo el planeta.

\footnotetext{
${ }^{21}$ Stephen D. Krasner, International Regimes, op. cit., p. 6.

${ }^{22}$ Ibid, p. 8.

${ }^{23}$ Ibid, p. 7.
} 
En su funcionamiento, el régimen de no proliferación nuclear descansa sobre el control de la oferta de tecnología, materiales y equipos nucleares ${ }^{24}$. Para ello, existen una serie de instrumentos jurídicos internacionales diseñados para restringir el acceso de los potenciales proliferadores a elementos tecnológicos que les pudieran servir para construir bombas o explosivos nucleares. Además del TNP, verdadero corazón del régimen, destacan el grupo de suministradores nucleares, el Comité Zangger, la Proliferation Security Initiative y el Acuerdo de Wassenaar. Aunque su función no es exactamente el control de la oferta de materiales, equipos y tecnología nuclear, hemos incluido una pequeña reseña de la Organización del Tratado de Prohibición Completa de Ensayos Nucleares, por su relación con las medidas de control de la oferta.

El TNP es uno de los tratados internacionales más exitosos: solo cinco Estados (India, Pakistán, Israel, Corea del Norte y Sudán del Sur) no son parte del TNP. En general, el éxito del TNP se debe en gran medida a las presiones de las superpotencias para imponer a los Estados de su bloque la política de no proliferación nuclear, con una combinación de coerción e incentivos. Es posible afirmar que el TNP ha tenido éxito en reducir la proliferación de armas nucleares (desde su creación, solo la India, Pakistán, Corea del Norte e Israel han accedido al arma nuclear, si bien Sudáfrica llegó a tener hasta seis cabezas nucleares, aunque las desmanteló posteriormente). No obstante, el TNP ha fracasado en su oferta de conseguir el desarme de los Estados nucleares.

En los años finales de la Guerra Fría, las dos superpotencias tomaron una serie de iniciativas para reducir sus respectivos arsenales nucleares. Así, se firmaron los tratados START ${ }^{25}$ y II $^{26}$, el citado Tratado INF o el Tratado $\mathrm{SORT}^{27}$. Estos acuerdos eran estrictamente bilaterales, y, aún después de su aplicación (y cada uno de ellos implicaba el desmantelamiento de miles de cabezas nucleares), los arsenales nucleares soviéticos y norteamericanos seguían siendo inmensamente superiores a los del resto de los Estados nucleares y, aún más, a los de los Estados que obtuvieron estas armas al margen del TNP.

\section{"El mundo nuevo" (nuclear)}

La caída del bloque socialista tuvo unas consecuencias geopolíticas de enorme calado. Por un lado, desaparece uno de los "polos" del sistema que pasa a tener una estructura unipolar, una situación sin antecedentes históricos.

\footnotetext{
${ }^{24}$ Christian Enemark, "Farewell to WMD: The Language and Science of Mass Destruction", en Contemporary Security Policy, vol. 32, no 2, de agosto de 2011, p. 393. Disponible en: http://www. tandfonline.com/doi/abs/10.1080/13523260.2011.590362?journalCode=fcsp20 3 de marzo de 2019.

${ }^{25}$ Tratado de Reducción de Armas Estratégicas o Strategic Arms Reduction Treaty firmado en 1991.

${ }^{26}$ Actualización del anterior, firmado en 1993.

${ }^{27}$ Tratado de Reducciones de Ofensivas Estratégicas o Strategic Offensive Reductions Treaty de 2002.
} 
Por otro, la ideología comunista, la única alternativa ideológica a la democracia liberal queda enormemente desprestigiada, por el fracaso económico y político de su principal valedora, la Unión Soviética. En el campo nuclear, esta situación se traduce en una repentina pérdida de utilidad del arma nuclear: de garantes absolutos de la disuasión (y, por ello, de la paz), las armas nucleares pasan a ser artefactos muy costosos sin una finalidad clara. ¿A quién disuadir tras el "fin de la historia"? En este nuevo escenario mundial, el poder militar convencionalde Estados Unidos le otorga una gran libertad de acción sin tener que recurrir al armamento nuclear (como confirmó la aplastante victoria sobre el Irak de Sadam Hussein en 1991). El armamento nuclear pierde interés en el mundo político y para la opinión pública. Desaparecida la amenaza de existencia global que representaba la MAD, hoy en día las cuestiones nucleares suscitan un escasísimo interés popular, como muestra el nulo interés público que se dedica a las sucesivas conferencias de revisión del TNP, incluso en este 2020 en el que se celebra el $50^{\circ}$ aniversario de su entrada en vigor.

El renacimiento de Rusia como potencia con aspiraciones globales y el auge de China hace que Estados Unidos haya dejado de ser el único "polo" del sistema. Nos acercamos a un mundo multipolar. Y, si de algo nos sirve la experiencia histórica, es posible defender que la estructura multipolar es temporal y, por ello, inestable.

$\mathrm{Y}$, pese a la aparente irrelevancia de las armas nucleares, vivimos en un "mundo nuclear" en el que todas las cuestiones relevantes de relación entre las grandes potencias están condicionadas por la existencia del arma nuclear. Más aún cuando Rusia solo tiene su arsenal nuclear como argumento para mantener su estatus de potencia mundial, y China tiene un arsenal manifiestamente inferior al de sus posibles rivales.

Es evidente que, tras la caída de la Unión Soviética, Rusia no ha podido heredar el papel de hegemón del conjunto de Estados que formaba el bloque socialista, y que Estados Unidos ha perdido gran parte de su capacidad de coerción frente a neutrales y aliados que tenía durante la Guerra Fría, más aún tras sus fracasos en la posguerra iraquí y en la ocupación de Afganistán, fracasos que han mostrado los límites del poder militar convencional norteamericano. Además de a la pérdida de influencia de las dos superpotencias "tradicionales", asistimos hoy al imparable incremento de poder de China, que emerge como una tercera superpotencia.

Así, como se ha citado, tras el final de la Guerra Fría, el mundo pasó de una situación de bipolaridad, a otra de unipolaridad y es posible afirmar que el sistema internacional se dirige ahora hacia una situación de multipolaridad.

En su clasificación de sistemas conforme al número de polos de poder -y compartiendo la citada definición de Snyder-, Mearsheimer ${ }^{28}$ distingue dos

28 John J. Mearsheimer, The Tragedy of Great Power Politics, Nueva York, W.W. Norton, 2003, pp. 334-347.

Araucaria. Revista Iberoamericana de Filosofia, Política, Humanidades y Relaciones Internacionales, año 22, $\mathrm{n}^{\circ} 44$. Segundo semestre de 2020. Pp. 427-448. ISSN 1575-6823 e-ISSN 2340-2199 https://dx.doi.org/10.12795/araucaria.2020.i44.20 
posibles casos para la multipolaridad que denomina multipolaridad equilibrada y multipolaridad desequilibrada. En el primero de estos casos, ninguna gran potencia destaca exageradamente sobre el resto (sería el caso del mundo aparecido tras la Paz de Westfalia o tras el Congreso de Viena). El segundo de ellos, la multipolaridad desequilibrada se da cuando un sistema compuesto por grandes potencias contiene un potencial hegemón (sería la situación del ejemplo propuesto por Deutsch y Singer ${ }^{29}$ ). En este caso, este hegemón en ciernes se siente con capacidad de alterar el equilibrio de poder incluso por la fuerza $\mathrm{y}$, al mismo tiempo, el temor que despierta tiende a suscitar una coalición antihegemónica (de acuerdo con el principio del equilibrio de poder, enunciado por Kenneth N. Waltz $)^{30}$ con un riesgo importante de generar un conflicto armado. Esta situación de multipolaridad desequilibrada sería la antesala de una unipolaridad (cuando una potencia en auge creciente aspira a ser hegemónica) o de una multipolaridad equilibrada (caso de una potencia hegemónica en decadencia). En este segundo caso, la potencia principal se vería obligada a mantener su reputación con recursos decrecientes, mientras que la cada vez menos desfavorable percepción de la comparación de capacidades militares disminuiría su poder de coerción (y, por tanto, las limitaciones que el sistema impone a los demás Estados) y alentaría nuevos desafíos. Un ejemplo histórico podría ser la España de los Austrias menores, periodo que culminó con la Guerra de los Treinta Años, la más destructiva librada en suelo europeo hasta las guerras napoleónicas.

Por otra parte, en los sistemas multipolares equilibrados, de forma similar al caso de los sistemas bipolares, las restricciones impuestas por la estructura serían relativamente importantes (cada superpotencia impondría límites a sus aliados y cada una de ellas restringiría además la libertad de acción de las otras), resultando un sistema más seguro ${ }^{31}$. De la misma forma, en un sistema multipolar equilibrado, las potencias estarían menos inclinadas a intervenir en todos los conflictos para mantener su reputación de superpotencia, al existir siempre otros candidatos susceptibles de intervenir en un caso concreto (como ejemplo, puede citarse el intento de Gran Bretaña, Francia o la Unión Soviética de evitar enfrentarse a la Alemania nazi, intentando que otra de las grandes potencias de la época asumiese el liderazgo en ese tema $)^{32}$.

La multipolaridad no implica necesariamente un entorno de seguridad más benigno para el resto de Estados "menores". Como en el ejemplo citado del Concierto de Europa, las grandes potencias pueden verse tentadas de establecer

${ }^{29}$ Deutsch y Singer, Multipolar Power Systems..., op. cit., p. 406.

${ }^{30}$ Kenneth N. Waltz, Theory of International Politics, Long Grove, Waveland Press Inc., 2010, (primera edición en 1979).

31 Shipin Tang, "A Systemic Theory of the Security Environment", en The Journal of Strategic Studies, vol. 27, n'1, de marzo de 2004, pp. 1-34, p.6.

32 Ibid, p. 61. 
un gobierno mundial, imponiendo sus intereses comunes al resto de Estados (véase la guerra de independencia griega, en el que las potencias europeas que formaban parte del Concierto de Europa decidieron que Grecia debía ser independiente, combatiendo y derrotando al Imperio otomano).

El fin de la bipolaridad implica también que los Estados no necesitan alinearse en un bloque. Pueden tener una relación más o menos próxima a Estados Unidos (la superpotencia restante) o a sus adversarios (Rusia y China), pero estas relaciones no implican el convertirse en enemigos existenciales de otros Estados. De la misma manera, al desaparecer el conflicto entre el mundo capitalista y el socialista, los conflictos locales y regionales han recuperado su autonomía, desligándose de la competición entre las superpotencias.

A diferencia de otras multipolaridades históricas, la existencia del arma nuclear implica la vigencia de la mencionada paradoja de la estabilidadinestabilidad: los polos del sistema (inevitablemente, Estados nucleares) saben que su arsenal nuclear es una salvaguarda de sus intereses vitales y que les permite desarrollar políticas agresivas frente a sus rivales, con la seguridad de que no arriesgan nada fundamental.

En consecuencia, en el campo específicamente nuclear, la bipolaridad reduciría la proliferación, mientras que la multipolaridad la favorecería ${ }^{33}$ : las razones para ello nacerían de que el final de la bipolaridad puso fin a muchas de las garantías de seguridad ${ }^{34}$ que las dos superpotencias otorgaban a sus aliados que eran un factor clave para limitar la proliferación nuclear, al tiempo que redujo la capacidad coercitiva de las superpotencias sobre el resto de Estados.

$\mathrm{Si}$ aceptamos como válida la multipolaridad desequilibrada como la estructura del sistema internacional en este nuevo mundo aparecido tras la Guerra Fría, quizá su principal característica sea que se trata de una estructura dinámica, con cambios continuos en la distribución de poder relativo entre las superpotencias, y por ello necesariamente inestable. En consecuencia, la creación de instituciones duraderas, como el conjunto de ellas que han dado forma a nuestro mundo entre el final de la Segunda Guerra Mundial y la actualidad, es extremadamente difícil, lo que nos llevará a un mundo mucho más anárquico y cambiante.

Sin embargo, la multipolaridad desequilibrada es una situación transitoria que deberá evolucionar hacia una nueva unipolaridad, una bipolaridad diferente (¿Estados Unidos-China?) o una multipolaridad equilibrada. Todas estas

33 Benjamin Frankel, "The Brooding Shadow: Systemic Incentives and Nuclear Weapons Proliferation", en Security Studies, vol. 2, n 3/4 de la primavera/verano de 1993, pp. 37-78, p. 37.

34 Estas garantías pueden entenderse como "los medios y métodos empleados para convencer a un aliado o socio de que Estados Unidos puede garantizar su seguridad en caso de intimidación, coerción o ataque por parte de un tercero". En Justin V. Anderson y Jeffrey A. Larsen, Extended Deterrence and Allied Assurance: Key Concepts and Current Challenges for U.S. Policy, INSS Occasional Paper 69, Colorado Springs, USAF Academy, USAF Institute for National Security Studies, septiembre 2013, p. 6.

Araucaria. Revista Iberoamericana de Filosofia, Política, Humanidades y Relaciones Internacionales, año 22, $\mathrm{n}^{\circ} 44$. Segundo semestre de 2020. Pp. 427-448. ISSN 1575-6823 e-ISSN 2340-2199 https://dx.doi.org/10.12795/araucaria.2020.i44.20 
posibilidades son más estables que la actual multipolaridad desequilibrada. Sin embargo, esta evolución será previsiblemente larga, y tiene pocas posibilidades de ser completamente pacífica. Es decir, el "mundo nuevo" todavía queda lejos en el tiempo.

\section{Los "monstruos del clarosocuro"}

En nuestro mundo actual parece que nos hemos olvidado de las armas nucleares y que estas son reliquias de una época felizmente superada. Sin embargo, en mi opinión, las armas nucleares estarán más presentes que nunca en el mundo al que nos dirigimos y el riesgo de una guerra nuclear (si bien, limitada) es mucho mayor que en el jerarquizado mundo de la Guerra Fría.

En efecto, la desaparición de la bipolaridad implica también el fin de la MAD: las dos superpotencias con arsenales nucleares capaces de destruir el mundo han dejado de ser una "amenaza existencial" la una para la otra, por lo que el riesgo de una guerra termonuclear que destruya el planeta se ha alejado mucho. Este hecho tiene múltiples consecuencias, entre las que cabe destacar una fundamental: el empleo del arma nuclear en un conflicto local o regional no implicaría necesariamente una guerra termonuclear global. Es decir, las consecuencias de un conflicto nuclear limitado geográficamente afectarían poco a aquellos Estados alejados del lugar del conflicto. Y, en consecuencia, los incentivos de estos Estados para inmiscuirse en un posible conflicto nuclear geográficamente alejado son mucho menores. Por ello, los beneficios del régimen de no proliferación nuclear ya no se perciben necesariamente como "universales" por todos los Estados. De la misma forma, si una guerra nuclear no implica necesariamente el fin de la vida en la Tierra (caso de un conflicto nuclear "limitado"), su uso respondería a un cálculo de coste/beneficio, por lo que es posible considerar un uso militar "real" del armamento nuclear.

Desaparecido el "conflicto existencial" característico de la Guerra Fría, los posibles conflictos armados a los que se enfrentarán las superpotencias actuales serán siempre limitados. Y, por ello, los sacrificios que estarán dispuestas a hacer serán igualmente $\operatorname{limitados}^{35}$. En consecuencia, incluso arsenales nucleares relativamente reducidos (al alcance de la mayoría de los Estados) serán efectivos como elemento de disuasión frente a todos los Estados del sistema internacional (incluso frente a las superpotencias). Y, por ello, la posesión de armamento nuclear será cada vez más una prioridad para los Estados enfrentados a alguna de las superpotencias. Los casos de Gadafi (Libia renunció al armamento nuclear en 2003 a cambio de "normalizar" sus relaciones con

\footnotetext{
${ }_{35}$ Andrew Mack, Why Big Nations Lose Small Wars: The Politics of Asymmetric Conflict, en World Politics, vol. 27, nº 2, enero de 1975, pp. 175-200.
} 
Occidente; en 2011, una coalición occidental derribó su régimen, y el dictador libio fue cruelmente asesinado por una turba) o de Ucrania (en el Tratado de Budapest de 1995, Ucrania accedió a entregar las armas nucleares presentes en su territorio, "herencia" de la Unión Soviética a Rusia, a cambio de que Estados Unidos, Reino Unido y la propia Rusia garantizasen su integridad territorial; en 2014, Rusia se anexionaba Crimea, y rebeldes apoyados por Rusia se apoderaron de la provincia de Donbass; las tibias reacciones de Estados Unidos y del Reino Unido no sirvieron para revertir la situación), o la diferencia de trato que reciben Irán (en proceso de obtener armas nucleares y sujeto a fuertes presiones y sanciones por parte norteamericana) y Corea del Norte (que ya dispone de ese armamento y que obtiene ayudas a cambio simplemente de acceder a negociar), Pakistán o la India, son incentivos para que los Estados que aspiran a modificar la distribución de poder en el sistema internacional se hagan con armamento nuclear. El caso de Pakistán y la India es muy especial en el sentido de que son Estados que han obtenido armamento nuclear al margen del régimen de no proliferación. Y, sin embargo, su condición ha sido aceptada por los Estados nucleares del TNP, lo que cuestiona la utilidad de la adhesión al régimen del resto de Estados no nucleares. En cualquier caso, si alguno (o algunos) de estos Estados descontentos con el actual statu quo consiguen desarrollar armas nucleares, sus rivales regionales también lo harán.

Por todo lo anterior, ni es previsible un desarme nuclear por parte de los Estados nucleares del TNP, ni el actual régimen de no proliferación nuclear podrá garantizar en el futuro a todos los Estados parte que sus rivales no accederán al armamento nuclear. De esta manera, dos de los compromisos esenciales en los que se basa el régimen de no proliferación nuclear quedan en entredicho.

El tercero de esos compromisos era la oferta de ayuda tecnológica para acceder a los usos pacíficos de la energía nuclear. De hecho, como se ha citado, el funcionamiento práctico del régimen se basa en restringir el acceso a la tecnología y a los materiales fisibles. Sin embargo, la tecnología nuclear tiene ya 80 años, por lo que cada vez más Estados tienen el nivel técnico suficiente como para desarrollar esa tecnología. Brasil, Turquía, Malasia, Indonesia, Taiwán son ahora suministradores de componentes nucleares. Por ello, al existir fuentes alternativas para acceder a la tecnología nuclear, la necesidad de asumir las renuncias que implica la pertenencia al régimen de no proliferación es mucho menos perentoria.

Como consecuencia de todo lo anterior, algunos Estados perciben beneficios mayores en desarrollar armas nucleares que en permanecer en el régimen de no proliferación nuclear. Y, en un efecto "bola de nieve", el acceso al arma nuclear de determinados Estados arrastrará a otros (caso muy probable de Arabia Saudí o Turquía si Irán accede al armamento nuclear, por ejemplo). 
Como es lógico, cuantas más armas nucleares haya, más decisores tengan la capacidad de emplearlas y más Estados (estables o no) tengan almacenadas armas nucleares (con grados de seguridad muy variables), la posibilidad de un error, de un accidente o de que estas armas acaben en manos de grupos terroristas será mayor.

Por último, conforme la memoria de los bombardeos de Hiroshima y Nagasaki y de la amenaza permanente de un holocausto nuclear en el que vivía la población mundial durante la Guerra Fría se vaya olvidando, el riesgo de que un líder (de un Estado o de un grupo no estatal) decida emplear el armamento nuclear será cada vez mayor.

\section{Conclusiones}

La estrategia nuclear no es un tema de interés histórico. Desgraciadamente, es una cuestión llamada a conocer una creciente actualidad. El régimen de no proliferación nuclear actual ha sido una herramienta fundamental para reducir el riesgo de una guerra nuclear. Sin embargo, es uno de los "hijos" de la Guerra Fría, y su diseño y operación corresponden a la situación del mundo en el momento de su creación, en términos de la distribución de poder entre los Estados (el elemento clave que determina la estructura del sistema internacional) y de las posibilidades tecnológicas de cada Estado en aquel momento. Conforme nos alejamos de ellas, sus limitaciones se hacen más evidentes, y su capacidad para cumplir sus fines disminuye.

La estructura actual del sistema internacional (multipolaridad desequilibrada) es inestable y, por ello, resulta muy difícil adoptar un nuevo régimen de no proliferación nuclear basado en la nueva distribución del poder y de las nuevas capacidades tecnológicas. Es indudable que estamos en un momento histórico y, si "la violencia es la partera de la historia", es difícil que la transición entre dos mundos tan distintos sea completamente pacífica. Vivimos en un mundo nuclear y, pese a la abundancia de buenas intenciones, seguiremos haciéndolo en el futuro. Por ello es imperativo refundar el régimen de no proliferación nuclear y adaptarlo a la nueva realidad del sistema internacional.

La alternativa es un riesgo muy elevado de sufrir una guerra nuclear de imprevisibles consecuencias. 


\section{Referencias bibligráficas:}

Anderson, Justin V. y Larsen, Jeffrey A., Extended Deterrence and Allied Assurance: Key Concepts and Current Challenges for U.S. Policy, INSS Occasional Paper 69, Colorado Springs, USAF Academy, USAF Institute for National Security Studies, septiembre 2013.

Brodie, Bernard S., The Absolute Weapon: Atomic Power and World Order, Nueva York, Harcourt Brace, 1946.

Correll, John T., "The Ups and Downs of Counterforce", en Air Force Magazine, octubre de 2005.

D’Olier, Franklin et alt., U.S. Strategic Bombing Survey, Washington, 1946 (reimpreso en 1987 por la USAF). Disponible en: http://web.archive.org/ web/20080528051903/http://aupress.au.af.mil/Books/USSB S/USSBS. pdf.

Deutsch, Karl W. y Singer, David J., "Multipolar Power Systems and International Stability", en World Politics, de la Universidad John Hopkins, vol. 16, n 3, de abril de 1964.

Enemark, Christian, "Farewell to WMD: The Language and Science of Mass Destruction", en Contemporary Security Policy, vol. 32, n 2 , de agosto de 2011. Disponible en: http://www.tandfonline.com/doi/abs/10.1080/13523 260.2011.590362? journalCode=fcsp20 3 de marzo de 2019.

Frankel, Benjamin, "The Brooding Shadow: Systemic Incentives and Nuclear Weapons Proliferation”, en Security Studies, vol. 2, no 3/4 de la primavera/ verano de 1993.

Goldschmdt, Bertrand, The Atomic Complex. A Worldwide Political History of Nuclear Energy. La Grange Park, American Nuclear Society, 1982.

Krasner, Stephen D., International Regimes, Ithaca, Cornell University Press, 1982.

Mack, Andrew, "Why Big Nations Lose Small Wars: The Politics of Asymmetric Conflict", en World Politics, vol. 27, n 2, enero de 1975.

Mandelbaum, Michael, The Nuclear Revolution: International politics before and after Hiroshima, Cambridge, University of Cambridge Press, 1981.

Mearsheimer, John J., The Tragedy of Great Power Politics, Nueva York, W.W. Norton, 2003.

Murdock, Clark A., "A Nuclear Deterrent for the 21st Century", en Craig Cohen y Josiane Gabel, Global Forecast 2015, Crisis and Opportunity, Washington, Center for Strategic and International Studies, 2014, p. 17. Disponible en: http://csis.org/files/publication/141110_Cohen_ GlobalForecast2015_Web.pdf 
Schelling, Thomas C. en Colby, Elbridge A. y Gerson, Michael S., Strategic Stability: Contending Interpretation, Carlisle, Strategic Studies Institute, febrero de 2013, p. v- Disponible en: http://www.strategicstudiesinstitute. army.mil/pdffiles/PUB1144.pdf 12 de febrero de 2020.

Snyder, Glenn H. and Diesing, Paul, Conflict among Nations: Bargaining, Decision Making and System Structure in International Crisis, Princeton, Princeton University Press, 1977.

Snyder, Glenn, "The Balance of Power and the Balance of Terror," en Seabury, Paul (ed.), The Balance of Power, San Francisco, Chandler, 1965.

Sokolski, D. (ed.), The Next Arms Race, Carlisle Barracks, Strategic Studies Institute, julio de 2012, p. 24. Disponible en: http://www. strategicstudiesinstitute.army.mil/pdffiles/PUB1113.pdf 8 de septiembre de 2019.

Tang, Shipin, "A Systemic Theory of the Security Environment", en The Journal of Strategic Studies, vol. 27, nº 1, de marzo de 2004.

Waltz, Kenneth N., Theory of International Politics, Long Grove, Waveland Press Inc., 2010, (primera edición en 1979).

Windrem, Robert, "North Korea likely lying about Hydrogen bomb test, experts say", en $N B C$ News.

Wohlstetter, Alfred et alt., Selection and Use of Strategic Air Bases, Santa Monica, RAND Corp. United States Air Force project RAND R-266, de abril de 1954 (reimpreso en 1962). Disponible en: http://www.rand.org/ content/dam/rand/pubs/reports/2006/R266.pdf 18 de febrero de 2020.

Wohlstetter, Alfred, "The Delicate Balance of Terror", Santa Monica, RAND Corporation, 6 de noviembre de 1958. Disponible en la página web de la RAND Corporation: http:/www.rand.org/about/history/wohlstetter/ P1472/P1472.html 20 de mayo de 2019. 